\title{
Carvacrol loaded electrospun fibrous films from zein and poly(lactic acid) for active food packaging
}

\author{
Aylin Altan ${ }^{\mathrm{a}, *}$, Zeynep Aytac ${ }^{\mathrm{b}}$, Tamer Uyar ${ }^{\mathrm{b}, * *}$ \\ ${ }^{a}$ Department of Food Engineering, Mersin University, Ciftlikkoy, Mersin 33343, Turkey \\ ${ }^{\mathrm{b}}$ Institute of Materials Science \& Nanotechnology, UNAM-National Nanotechnology Research Center, Bilkent University, Ankara 06800, Turkey
}

\section{A R T I C L E I N F O}

Article history:

Available online 17 February 2018

\section{Keywords:}

Electrospinning

Zein

PLA

Carvacrol

Bread

Active packaging

\begin{abstract}
A B S T R A C T
The composite fibrous films were developed from zein and poly(lactic acid) (PLA) by incorporating carvacrol at three different concentrations (5, 10 and 20\%) using electrospinning. The morphology and size of fibers obtained from both zein and PLA were affected by the level of the incorporated carvacrol. The Fourier transform infrared (FTIR) spectroscopy and thermogravimetric analysis (TGA) results showed that carvacrol was encapsulated in electrospun zein and PLA fibers. The antioxidant activity of carvacrol loaded zein fibers ranged from 62 to $75 \%$, while antioxidant capacity of PLA fibers varied from 53 to $65 \%$ for $5-20 \%$ carvacrol content. The composite fibrous films showed a sustained diffusion controlled release behavior. Preliminary studies on whole wheat bread samples showed that carvacrol loaded electrospun zein and PLA fibers are able to preserve bread samples, indicating that they are good candidates for active food packaging applications to extend the shelf life of whole wheat bread.
\end{abstract}

() 2018 Elsevier Ltd. All rights reserved.

\section{Introduction}

Electrospinning is an efficient and cost-effective technique for the production of fibers from both natural and synthetic polymers. This technique involves the stretching of a polymer solution in a high electric field to produce fibers with a diameter ranging from tens of nanometer to submicron. Electrospun fibers exhibit a large surface area to mass ratio and their surface can be functionalized. These properties have led to diverse applications of electrospun nanofibers in tissue engineering, drug delivery systems, biosensors and many others (Uyar \& Kny, 2017). Most recently, there has been an increasing interest for the potential of electrospinning in the field of food science as an alternative method for encapsulation of bioactive compounds (Drosou, Krokida, \& Biliaderis, 2017). In food processing and packaging, electrospun fibers have been proposed for stabilizing or controlling the release of the active compounds (Kayaci \& Uyar, 2012; Li, Lim, \& Kakuda, 2009; Vega-Lugo \& Lim, 2009). However, the limited number of studies are present for the application of electrospinning in active packaging (Aytac, Keskin, Tekinay, \& Uyar, 2017; Wen et al., 2016).

\footnotetext{
* Corresponding author.

** Corresponding author.

E-mail addresses: aaltan@mersin.edu.tr, aylinaltan@yahoo.com (A. Altan), tamer@unam.bilkent.edu.tr (T. Uyar).
}

Active packaging is defined as a type of packaging that changes the condition of the packaging to prolong the shelf life or enhance safety or sensory properties while maintaining the quality of the food (Talens, Fabra, \& Chiralt, 2011). The incorporation of antimicrobial substances in packaging materials is a promising way to produce active packaging materials and to control undesirable growth of microorganisms (Vermeiren, Devlieghere, van Beest, de Kruijf, \& Debevere, 1999). Natural antimicrobial agents such as essential oils have attracted increasing interest due to natural antifungal, insecticidal, antimicrobial and antioxidant properties. Carvacrol is a major component of oregano and thyme oil (Wu, Luo, \& Wang, 2012). It contains phenolic compounds that possess the antimicrobial properties against food-borne pathogens, fungi, yeast and molds (López, Sáncez, Battle, \& Nerín, 2007; Nostro et al., 2012; Quintero, Rodriguez, Bruna, Guarda, \& Galotto, 2013; Ramos et al., 2013). Carvacrol has been recently incorporated in chitosan nanoparticles, cellulose acetate, polypropylene, polyethylene-covinylacetate and sodium-calcium caseinate films (Arrieta, Peltzer, Garrigós, \& Jiménez, 2013; Keawchaoon \& Yoksan, 2011; Nostro et al., 2012; Quintero et al., 2013; Ramos, Jiménez, Peltzer, \& Garrigós, 2012). To the best of our knowledge, the loading of carvacrol into electrospun fibers from zein and poly (lactic acid) (PLA) has not been reported.

Zein is a water insoluble hydrophobic storage protein in corn kernel and is renewable and biodegradable. Zein has natural water 
and grease resistant properties since it is hydrophobic due to presence of the apolar amino acids such as proline, leucine and glutamine in it (Shukla \& Cheryan, 2001; Torres-Giner, Ocio, \& Lagaron, 2009; Wang \& Chen, 2012). PLA is a biodegradable, compostable and biocompatible polymer and safe for the environment and for the food packaging applications (Rhim, 2013). Therefore, the objectives of this study were to incorporate carvacrol in electrospun zein and PLA fibers and evaluate its impact on fiber characteristics and to determine release behavior of carvacrol from composite fibrous membrane. Preliminary studies were also conducted on whole wheat bread as a model food to determine effectiveness of obtained fibers for preserving fresh food.

\section{Materials and methods}

\subsection{Materials}

Zein from maize, carvacrol (CRV) (98\%), 2,2-diphenyl-1picrylhydrazyl (DPPH), chloroform (99-99.4\%) and ethanol (99.8\%) were purchased from Sigma-Aldrich. PLA (4043D) was kindly donated by NatureWorks LLC Co. N,N-Dimethyl formamide (DMF) was supplied by Merck.

\subsection{Preparation of polymer solutions}

Zein solution ( $30 \% \mathrm{w} / \mathrm{v}$ ) was prepared by dissolving zein powder in aqueous ethanol $(80 \% \mathrm{v} / \mathrm{v})$ solution. PLA $(10 \% \mathrm{w} / \mathrm{v})$ was dissolved in a chloroform/DMF $(9: 1, v / v)$ solvent system. Both solutions were stirred at room temperature under magnetic stirring until polymers completely dissolved. Carvacrol (CRV) was added at levels of 5, 10 and $20 \%(\mathrm{w} / \mathrm{w})$ with respect to polymer content. After mixing carvacrol for $30 \mathrm{~min}$ at room temperature, solutions were used for electrospinning.

\subsection{Rheological measurements}

The rheological measurements were determined by Haake Rheostress RS1 rheometer (Karslruhe, Germany) equipped with a plate-plate accessory (diameter $35 \mathrm{~mm}$, gap $1 \mathrm{~mm}$ ) at a controlled shear rate between 0 and $500 \mathrm{~s}^{-1}$ at $25^{\circ} \mathrm{C}$. The flow curves of zein and PLA solutions obtained from shear stress versus shear rate data were fitted to the power-law equation:

$\tau=K \dot{\gamma}^{n}$

where $\tau$ is the shear stress (Pa), $\dot{\gamma}$ is the shear rate $\left(\mathrm{s}^{-1}\right), K$ is the consistency coefficient and $n$ is the flow behavior index. The apparent viscosity of PLA solutions was determined using the power-law equation for viscosity (Eq. (2)) at a shear rate of $100 \mathrm{~s}^{-1}$.

$\eta=K \dot{\gamma}^{n-1}$

where $\eta$ is the apparent viscosity (Pa s). Experiments were performed in triplicate. The rheological properties of solutions are given in Table 1.

\subsection{Electrospinning process}

A $5 \mathrm{~mL}$ syringe fitted with a metallic needle $(0.7 \mathrm{~mm}$ outer diameter) was used for electrospinning of zein and PLA solutions. The syringe was mounted on a syringe pump (KDS 101, KD Scientific). Polymer solutions were electrospun using the horizontal set up. The solution flow rate was kept constant at $1 \mathrm{~mL} / \mathrm{h}$ for both polymer solutions. The applied voltage was adjusted to $15 \mathrm{kV}$ by a high voltage power supply (Spellman, SL Series). The distance between the collector and needle tip was $20 \mathrm{~cm}$. Fibers were collected on a grounded metal collector covered by aluminum foil. The electrospinning unit was placed in Plexiglas box and electrospinning experiments was carried out at $24{ }^{\circ} \mathrm{C}$ and $21 \%$ relative humidity.

\subsection{Characterization}

The morphology of electrospun zein and PLA fibers was examined by a field emission scanning electron microscope (FESEM) (Supra 55, Carl Zeiss, Germany) at an operating voltage of $5 \mathrm{kV}$. The samples were mounted on metal stubs using a double-sided adhesive tape and coated with $2 \mathrm{~nm}$ platinum prior to examination (Q150R ES, Quorum Technologies, England). In order to determine average fiber diameter, 100 fibers were analyzed using image analysis software (Image J, NIH, Maryland, USA).

For Fourier transform infrared (FTIR) spectroscopy analyses, a small amount of sample was mixed with potassium bromide $(\mathrm{KBr}$, FTIR grade) in a mortar, and then a pellet was obtained using a press by applying high pressure. The infrared spectra of the samples were obtained by using FTIR spectroscopy (Bruker-VERTEX 70). The FTIR spectra were recorded from 400 to $4000 \mathrm{~cm}^{-1}$, at a resolution of $4 \mathrm{~cm}^{-1}$, by taking 64 scans for each sample.

The thermal stability of the zein and PLA fibers was investigated by thermogravimetric analysis (TGA; TA Q500, USA). The measurements were performed under nitrogen atmosphere, and the samples were heated up to $450^{\circ} \mathrm{C}$ at a constant heating rate of $20^{\circ} \mathrm{C} / \mathrm{min}$.

\subsection{Release study}

The cumulative amount of carvacrol released from zein and PLA fibers were determined at 160 and $350 \mathrm{~min}$ by headspace gas chromatography-mass spectrometry (GC-MS) of Agilent Technologies 7890A gas chromatograph coupled to an Agilent Technologies $5975 \mathrm{C}$ inert MSD with a triple-axis detector. The used capillary column was HP-5MS (Hewlett-Packard, Avondale, PA) ( $30 \mathrm{~m} \times 0.25 \mathrm{~mm}$ i.d., $0.25 \mu \mathrm{m}$ film thickness). The headspace GCMS experiments were performed with a CTCPAL auto sampler. Electrospun fiber of zein and PLA fiber (14 mg) was placed in $20 \mathrm{~mL}$ headspace glass vials. The vials were agitated at $500 \mathrm{rpm}$ at $30^{\circ} \mathrm{C}$ of incubation temperature. Helium was used as carrier gas was at a flow rate of $1.2 \mathrm{~mL} / \mathrm{min} 500 \mu \mathrm{L}$ of vapor was injected to the headspace GC-MS by using a headspace injector (MSH 02-00B, volume: $2.5 \mathrm{~mL}$, scale: $60 \mathrm{~mm}$ ). The syringe temperature was $35^{\circ} \mathrm{C}$. Oven temperature was held at $75^{\circ} \mathrm{C}$ for $1 \mathrm{~min}$ and increased to $250^{\circ} \mathrm{C}$ at the rate of $20^{\circ} \mathrm{C} / \mathrm{min}$ and held at this temperature for $5 \mathrm{~min}$. Thermal desorption was conducted in the split mode (20:1). Headspace GC-MS analyses were carried out in the complete selected ion monitoring mode (SIM). Flavor 2 and NIST 0.5 libraries were used to decide carvacrol peak. The release experiment was performed in triplicate and the results were reported as average \pm standard deviation

\subsection{Antioxidant activity}

The antioxidant activity of carvacrol loaded zein and PLA fibers was measured using 2,2-diphenyl-1-picrylhydrazyl (DPPH) radical. For the DPPH assay, $5 \mathrm{mg}$ of the fiber sample was mixed with $3 \mathrm{~mL}$ of DPPH solution $\left(10^{-4} \mathrm{M}\right)$ and allowed to stand in the dark for $30 \mathrm{~min}$ at room temperature. At the end of the $30 \mathrm{~min}$, the absorbance of the solution was measured with UV-Vis NIR Spectroscopy (Varian Cary 5000) at $517 \mathrm{~nm}$. The antioxidant activity (AA) of samples was calculated using the following equation: 
Table 1

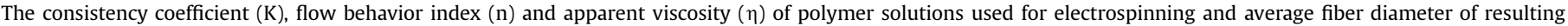
electrospun fibers.

\begin{tabular}{|c|c|c|c|c|c|c|}
\hline Polymer & $\begin{array}{l}\text { Carvacrol content } \\
(\%, w / w)\end{array}$ & $\begin{array}{l}\mathrm{K} \\
\left(\mathrm{Pa} \mathrm{s}^{\mathrm{n}}\right)\end{array}$ & $\mathrm{n}$ & $\mathrm{R}^{2}$ & $\begin{array}{l}\eta \\
(\text { Pa s) }\end{array}$ & $\begin{array}{l}\text { Average fiber diameter } \\
(\mathrm{nm})\end{array}$ \\
\hline \multirow[t]{4}{*}{ Zein } & 0 & $0.156 \pm 0.003^{b}$ & $1.000 \pm 0.005^{\mathrm{a}}$ & 0.999 & - & $604 \pm 120^{b}$ \\
\hline & 5 & $0.154 \pm 0.005^{\mathrm{b}}$ & $1.000 \pm 0.006^{\mathrm{a}}$ & 0.998 & - & $647 \pm 113^{c}$ \\
\hline & 10 & $0.136 \pm 0.012^{\mathrm{a}}$ & $1.000 \pm 0.001^{\mathrm{a}}$ & 0.998 & - & $539 \pm 103^{a}$ \\
\hline & 20 & $0.137 \pm 0.002^{\mathrm{a}}$ & $1.000 \pm 0.002^{\mathrm{a}}$ & 0.999 & - & $553 \pm 123^{a}$ \\
\hline \multirow[t]{4}{*}{ PLA } & 0 & $2.502 \pm 0.094^{\mathrm{a}}$ & $0.903 \pm 0.001^{\mathrm{a}}$ & 0.997 & $1.600 \pm 0.067^{\mathrm{a}}$ & $1899 \pm 278^{\mathrm{a}}$ \\
\hline & 5 & $2.368 \pm 0.105^{\mathrm{a}}$ & $0.933 \pm 0.010^{\mathrm{b}}$ & 0.998 & $1.738 \pm 0.008^{\mathrm{a}}$ & $1822 \pm 500^{\mathrm{a}}$ \\
\hline & 10 & $2.706 \pm 0.020^{\mathrm{a}}$ & $0.925 \pm 0.011^{b}$ & 0.998 & $1.922 \pm 0.114^{\mathrm{a}}$ & $1914 \pm 468^{a}$ \\
\hline & 20 & $4.047 \pm 0.410^{\mathrm{b}}$ & $0.920 \pm 0.002^{b}$ & 0.999 & $2.208 \pm 0.300^{\mathrm{b}}$ & $2268 \pm 395^{b}$ \\
\hline
\end{tabular}

$\mathrm{AA}(\%)=\frac{\left(\mathrm{A}_{\text {control }}-\mathrm{A}_{\text {sample }}\right)}{\mathrm{A}_{\text {sample }}} \times 100$

where $A_{\text {control }}$ and $A_{\text {sample }}$ are the absorbance values of the DPPH solution without and with the presence of the sample solutions. The measurements were carried out in triplicate.

\subsection{Study of effectiveness of the fibers to preserve bread}

Whole wheat bread without preservative was produced in the Center of Food Researches and Applications at the Mersin University, Mersin (Turkey). Preliminary studies were carried out to test the effectiveness of the zein and PLA fibers with carvacrol by putting them in petri dishes with sliced bread and further observing the occurrence of fungal growth on bread samples with time. For this purpose, bread slices (around $10 \mathrm{~g}$ ) were placed on the base of glass petri dishes (inside dimensions: $10.5 \mathrm{~mm}$ diameter, $2.5 \mathrm{~mm}$ height). Fibers ( $25-40 \mathrm{mg}$ ) were cut and placed in the petri dishes. There was no direct contact between fiber and bread samples. Petri dishes were sealed with parafilm and incubated at $25^{\circ} \mathrm{C}$ in an incubator during 7 days. Experiments were carried out in duplicate. After 7 days, microbiological quality of bread samples was evaluated by determining total aerobic and yeast and mold counts. Bread samples were homogenized with $90 \mathrm{~mL}$ of $0.1 \%$ peptone water for $30 \mathrm{~min}$ on a magnetic stirrer. Serial dilutions $\left(10^{-1}-10^{-6}\right)$ were performed using peptone water and $0.1 \mathrm{~mL}$ of dilutions was plated onto plate count agar for a total aerobic count and potato dextrose agar for yeast and mold count. The plates were incubated at $37^{\circ} \mathrm{C}$ for $24 \mathrm{~h}$ and at $25^{\circ} \mathrm{C}$ for $48-72 \mathrm{~h}$ for aerobic count and yeast and mold count, respectively (Lopes, Soares, Lopes, Silva, \& Júnior, 2014). The results are expressed in colony forming unit $\left(\right.$ CFU.g $\left.{ }^{-1}\right)$. The tests were performed in duplicated. The growth inhibition rate (\%) was calculated as follows:

Growth inhibition rate $(\%)=\frac{\left(\mathrm{C}_{\text {control bread }}-\mathrm{C}_{\text {sample }}\right)}{\mathrm{C}_{\text {control bread }}} \times 100$

where $\mathrm{C}_{\text {control bread }}$ and $\mathrm{C}_{\text {sample }}$ are the numbers of colonies $\left(\mathrm{CFU} . \mathrm{g}^{-1}\right.$ ) of the bread sample without fibers and with fibers stored at $25^{\circ} \mathrm{C}$ for 7 days.

\subsection{Data analysis}

Duncan's multiple range test was performed to determine the effect of carvacrol level on rheological parameters of polymer solutions, fiber characteristics and antioxidant activity by using SPSS 11.0 (SPSS Inc., Chicago, IL, USA). The parameters of the model used in the release study were calculated using the NLIN procedure of the Sigma Plot software (Scientific Graph System, version 10.0, SPSS Inc., Chicago, IL).

\section{Results and discussion}

\subsection{Characterization of electrospun fibers}

The effect of carvacrol content on the morphological structures of electrospun zein fibers is shown in Fig. 1. FE-SEM images showed that neat and carvacrol loaded zein fibers had ribbon-like morphology (Fig. 1a-d). The formation of ribbon-like morphology is due to rapid vaporization of ethanol from the surface of the jet and hence collapsing the resulted fibers. When the jet is collapsed, the diametrically opposite parts of the fiber skin come into contact and small tubes are formed at each edge of the ribbon (Koombhongse, Liu, \& Reneker, 2001; Neo, Ray, Easteal, Nikolaidis, \& Quek, 2012). The average diameter of pure zein fibers was $604 \pm 120 \mathrm{~nm}$, whereas those of zein fibers containing 5,10 and 20\% carvacrol were $647 \pm 113,539 \pm 103$ and $553 \pm 123 \mathrm{~nm}$, respectively. At 5\% carvacrol content, the average fiber diameter of zein fibers slightly changed when compared to the pure zein fibers. However, the average fiber diameter decreased significantly $(\mathrm{p}<0.05)$ when the carvacrol content was increased from 5 to $20 \%$ (Table 1). The change in fiber diameter distribution by the addition of carvacrol is also shown by histogram graph of FE-SEM images (Fig. 1). As shown in histogram graphs, the incorporation of carvacrol in the zein solutions resulted in narrower fiber diameter with homogeneous size distributions. The reduction of fiber diameter might be due to the increased charged density by the incorporation of carvacrol above 5\% level. Increasing charge density would improve the stretching forces and self-repulsion of the jet and thus it would decrease the fiber diameter (El-Naggar, Abdelgawad, Salas, \& Rojas, 2016). Another possible reason may be the effect of carvacrol level on viscosity of zein solutions. In order to evaluate the effect of carvacrol level on rheological properties of zein solutions, the experimental shear stress data as a function of shear rate were fitted to the power-law model (Eq. (1)). It was found that the experimental data fitted well with a coefficient of determination $\left(R^{2}\right)$ greater than 0.99 . The consistency coefficient $(K)$ and flow behavior index $(n)$ values are given in Table 1 . The flow behavior index values $(n=1)$ indicated that zein solutions with and without carvacrol showed Newtonian behavior. As carvacrol content increased from 5 to $20 \%$, the consistency coefficient decreased significantly $(\mathrm{p}<0.05)$ and thus viscosity of solutions decreased. The decrease in viscosity caused thinner fiber resulting from the higher degree of jet stretching during electrospinning (Neo et al., 2012). It is well known that solution parameters such as viscosity and electrical conductivity directly affect the shape and size of nanofibers (Torres-Giner, Gimenez, \& Lagaron, 2008).

The FE-SEM images of PLA fibers with different carvacrol content and their corresponding histogram graphs are illustrated in 

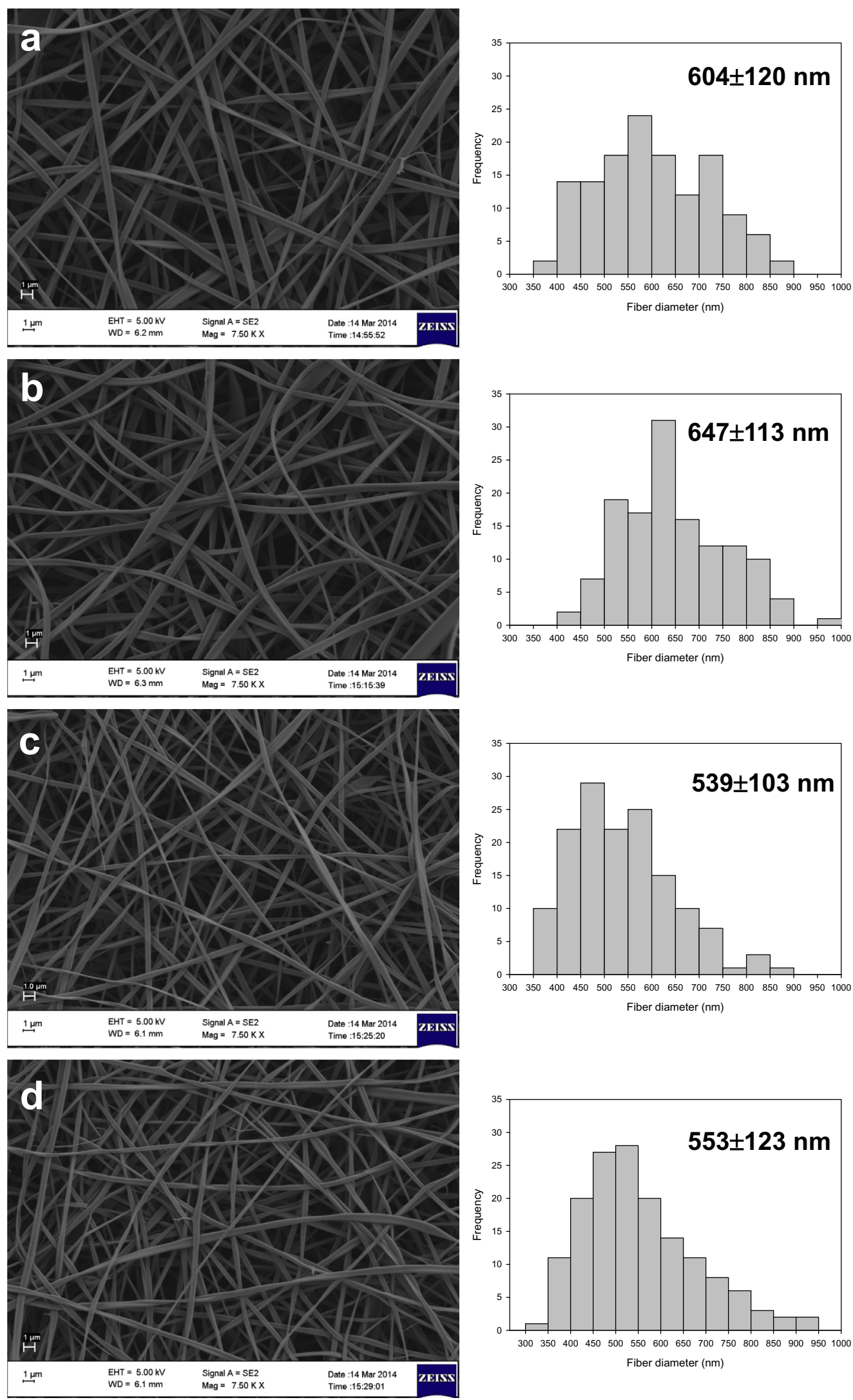

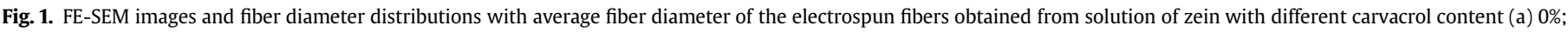
(b) $5 \%$; (c) $10 \%$; (d) $20 \%(\mathrm{w} / \mathrm{w})$. 
Fig. 2. The carvacrol free PLA fibers were smooth on surface and uniform with an average diameter of $1899 \pm 278 \mathrm{~nm}$. The average fiber diameter of fibers containing 5, 10 and $20 \%$ carvacrol was found as $1822 \pm 500,1914 \pm 468$ and $2268 \pm 395 \mathrm{~nm}$, respectively. The average fiber diameter increased significantly $(\mathrm{p}<0.05)$ as the carvacrol content was increased from 5 to $20 \%$ (Table 1). The increase in the average fiber diameter can be due to the increase of viscosity of PLA solutions with increasing carvacrol amount. The apparent viscosity values of PLA solutions are shown in Table 1 . The apparent viscosity increased with the addition of carvacrol to the PLA solution. However, the increase of apparent viscosity was only significant $(\mathrm{p}<0.05)$ for $20 \%$ carvacrol level. A higher viscosity would cause less stretching of jet, thereby reducing jet path and producing larger fibers (Phiriyawirut \& Phaechamud, 2012). The flow behavior index values of PLA solutions with and without carvacrol were lower than 1 , indicating a non-Newtonian shear thinning behavior (Table 1). This shear thinning behavior may improve polymer chain entanglement (Kriegel, Kit, McClements, \& Weiss, 2009), resulting in larger fiber diameter. In the study of Neo et al. (2013a), the increase in electrospun fiber diameter was attributed to the increased number of molecular entanglement in the solution. Khalifa (2015, p. 46) explained that such chain entanglement creates more resistance to the stretching of the polymer jet by the effect of electrostatic repulsive force during electrospinning.

The size of fibers varied unevenly by the incorporation of carvacrol (Fig. 2b-d). A fused morphology was observed for PLA fibers at $20 \%$ carvacrol content. Similar result was found by Zhang, Huang, Kusmartseva, Thomas, and Mele (2017). In this study, two or more PLA fibers had fused together and formed bundles at $10 \%(\mathrm{v} / \mathrm{v})$ of tea tree essential oil concentration in PLA solution. When the concentration of essential oil reached $15 \%$, highly interconnected fibers were produced. This kind of changes observed in PLA fiber morphology was attributed to the thermal properties of the composite fibers. Fig. 3 shows FE-SEM images of carvacrol loaded zein and PLA fibers at higher magnifications. Fibers electrospun from zein solutions containing different amount of carvacrol had a smooth surface morphology without any pores and pits (Fig. 3a-d). In the case of PLA fibers, the fiber structure changed from smooth to textured topography as the carvacrol content increased from 5 to $20 \%$ (Fig. 3e-h). At 10\% carvacrol content, PLA fibers showed an increase in surface roughness with few pits when compared with pure PLA fibers and PLA fibers containing 5\% carvacrol. Incorporation of carvacrol in the PLA solutions at $20 \%$ resulted in deep pits and wrinkles on the surfaces of PLA fibers (Fig. $3 \mathrm{~h}$ ). The volatile nature of carvacrol might affect solvent evaporation rate and hence caused pits on the surface of fiber. The reason of wrinkled surface occurred can be attributed to the shrinkage of the polymer jet resulting from the formation of interior pores (Liu, Huang, \& Jin, 2015).

The infrared spectra of the carvacrol, electrospun pure zein fiber and carvacrol loaded electrospun zein fibers is shown in Fig. 4a. The spectrum of carvacrol showed the stretching absorption peak at $3370 \mathrm{~cm}^{-1}$ associated with $-\mathrm{OH}$ group (Keawchaoon \& Yoksan, 2011). Peak at $2961 \mathrm{~cm}^{-1}$ could be attributed to $\mathrm{C}-\mathrm{H}$ stretching bonds. The bands between 1620 and $1420 \mathrm{~cm}^{-1}$ could be assigned to $\mathrm{C}-\mathrm{C}$ stretching of aromatic ring of carvacrol molecule. The $\mathrm{C}-\mathrm{O}$ stretching in $\mathrm{C}-\mathrm{OH}$ peak was observed around at $1252 \mathrm{~cm}^{-1}$ (Arrieta et al., 2013). The $\mathrm{C}=\mathrm{C}$ stretching of aromatic ring was found at 866 and $813 \mathrm{~cm}^{-1}$ (Arrieta et al., 2013; Keawchaoon \& Yoksan, 2011). Characteristic protein bands for pure zein fiber were found at 3413,1655 and $1542 \mathrm{~cm}^{-1}$ indicated the presence of amide A (N$\mathrm{H}$ stretching), amide I (C-O stretching) and amide II ( $\mathrm{N}-\mathrm{H}$ bending and C-N stretching), respectively (Arcan \& Yemenicioğlu, 2013; Wongsasulak, Tongsin, Intasanta, \& Yoovidhya, 2010). FTIR spectra of carvacrol loaded zein fibers showed that no new peaks appeared by the addition of carvacrol into zein solution. Although the FTIR spectra of carvacrol loaded electrospun zein fibers was similar to that of zein fibers, the characteristic peak of $\mathrm{N}-\mathrm{H}$ stretching of pure zein fiber at $3413 \mathrm{~cm}^{-1}$ shifted to 3436,3435 and $3437 \mathrm{~cm}^{-1}$ for 5,10 and $20 \%$ carvacrol loaded zein fibers, respectively. This peak shift observed in the $\mathrm{N}-\mathrm{H}$ band could be attributed to the interaction between phenolics of carvacrol and zein (Fuenmayor \& Cosio, 2016; Neo et al., 2013a). Fuenmayor and Cosio (2016) observed the changes in the N-H and amide bands by the incorporation of gallic acid in the zein fibers. Arcan and Yemenicioglu (2013) found a band shift in the amide A band with the addition of catechin in the zein film. They suggested that a band shift was due to the potential roles of H-bonds formed between zein and catechin. The $\mathrm{C}-\mathrm{C}$ and $\mathrm{C}-\mathrm{O}$ stretching peaks of carvacrol molecule at $1620-1420$ and $1252 \mathrm{~cm}^{-1}$ overlapped with electrospun zein fiber peak at 1655,1542 and $1252 \mathrm{~cm}^{-1}$, respectively. Therefore, the characteristic peaks of carvacrol were not obvious in the FTIR spectra of carvacrol loaded electrospun zein fibers.

Fig. $4 \mathrm{~b}$ represents the FTIR spectra of pure electrospun PLA fibers and carvacrol loaded PLA fibers. A broad peak around $3495 \mathrm{~cm}^{-1}$ could be assigned to the stretching of $\mathrm{O}-\mathrm{H}$ group of pure PLA. The PLA spectrum showed a peak at $1759 \mathrm{~cm}^{-1}$ related to the $\mathrm{C}=\mathrm{O}$ stretching of carbonyl groups of PLA. The peaks at 1184 and 1088 were attributed to $\mathrm{C}-\mathrm{O}-\mathrm{C}$ bending vibrations (Cosme, Silva, Nunes, \& Picciani, 2016) while peak at $1047 \mathrm{~cm}^{-1}$ was due to $\mathrm{C}-\mathrm{CH}_{3}$ vibrations (Armentano et al., 2015). The absorption bands at 1455 and $1384 \mathrm{~cm}^{-1}$ were observed. These peaks were attributed to the $\mathrm{C}-\mathrm{H}$ deformation from $-\mathrm{CH}_{2}$ (Cosme et al., 2016). The C-C stretching vibrations were identified at 870 and $757 \mathrm{~cm}^{-1}$ (Oliveira, Mattoso, Orts, \& Medeiros, 2013). Dai and Lim (2015) found absorption bands at 862 and $755 \mathrm{~cm}^{-1}$, which related to the amorphous and crystalline structure of PLA. The peak position at $3495 \mathrm{~cm}^{-1}$ shifted to around $3500 \mathrm{~cm}^{-1}$ in PLA fibers as carvacrol content increased from 0 to $20 \%$. The peak of carvacrol at $1620 \mathrm{~cm}^{-1}$ appeared in carvacrol loaded PLA fiber at $1637 \mathrm{~cm}^{-1}$. The intensity of this peak increased when carvacrol content increased from 5 to 20\% in PLA fibers. Similarly, the intensity of peaks at 1455, 1384, 870 and $757 \mathrm{~cm}^{-1}$ increased in carvacrol loaded PLA fibers. Bands of carvacrol molecule at 1459, 1383, 866 and $813 \mathrm{~cm}^{-1}$ were observed in spectrum of carvacrol loaded PLA fibers at 1455, 1384, 870 and $757 \mathrm{~cm}^{-1}$, respectively. The peak shifts suggested that the incorporation of carvacrol in the electrospun PLA fibers. In addition, the intensity of these peaks increased as carvacrol content increased, which shows presence of carvacrol in PLA fibers.

TGA was used to study the effect of carvacrol incorporation on thermal stabilities of the electrospun zein and PLA fibers. Fig. 5a shows TGA thermograms of carvacrol, electrospun pure zein and carvacrol loaded electrospun zein fibers. The TGA curve of pure zein fiber exhibited two stages of weight loss as a function of temperature. The first stage occurred in the range of $130-220^{\circ} \mathrm{C}$ with a weight loss of around $3.4 \%$, whereas the second stage was observed between 240 and $400{ }^{\circ} \mathrm{C}$. Both of these weight losses were attributed to the main degradation of zein, which is observed relatively slow at the beginning as reported in the study of Neo et al. (2013a). As shown in Fig. 5a, pure carvacrol evaporated completely at around $170^{\circ} \mathrm{C}$. Zein fibers showed one more stage of weight loss between 75 and $150^{\circ} \mathrm{C}$ after incorporation of carvacrol. The weight loss of carvacrol was calculated as $\sim 3.3,6.1$, and $9.2 \%$ for 5,10 and $20 \%$ carvacrol, indicating that indirect confirmation of presence of carvacrol in the zein fibers.

The peak temperatures at the highest rate of weight loss were found as 329,330 and $326^{\circ} \mathrm{C}$ for carvacrol loaded zein fibers at 5, 10 and $20 \%$ carvacrol level. These results showed that incorporation of carvacrol in the zein did not affect significantly thermal 

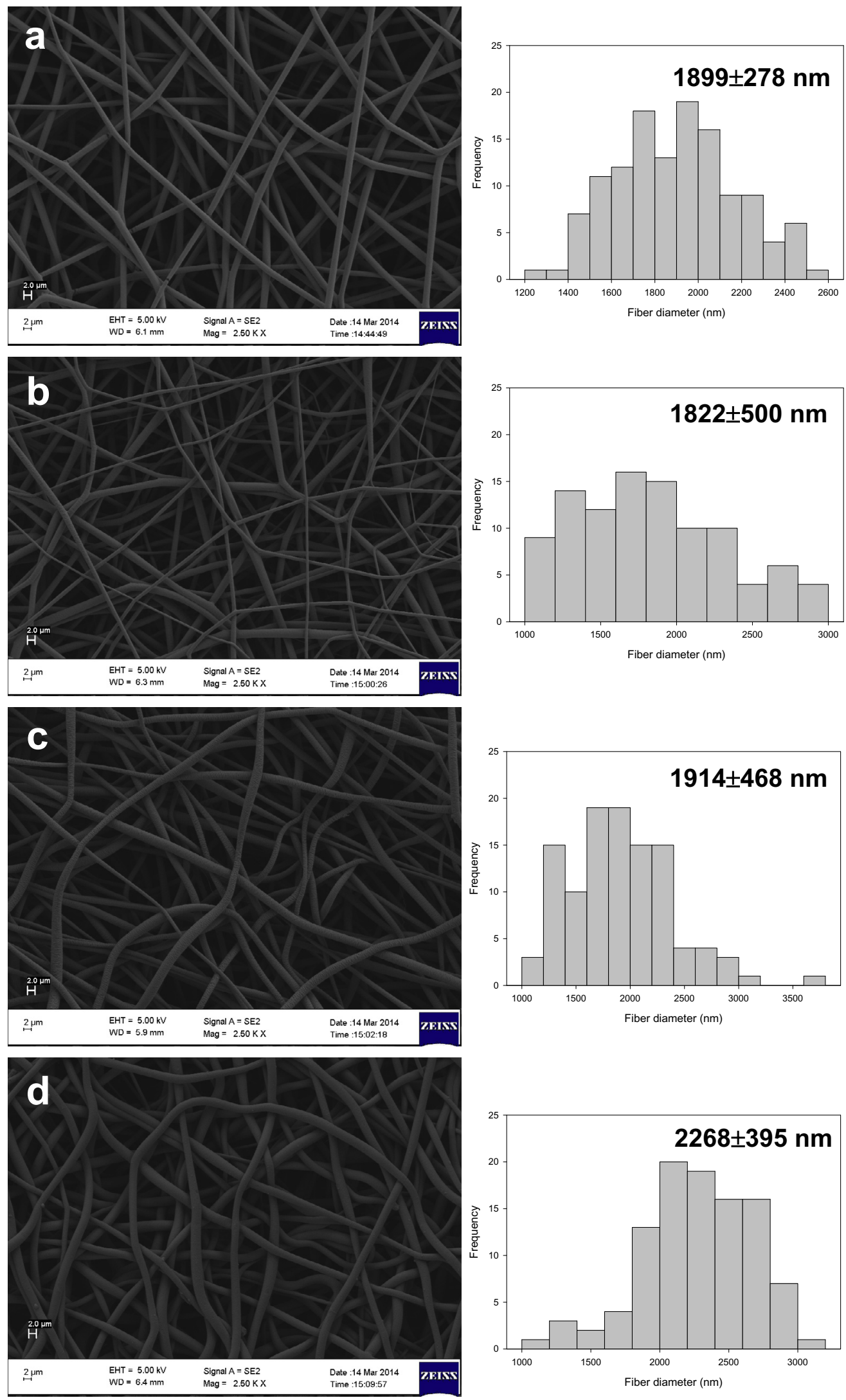

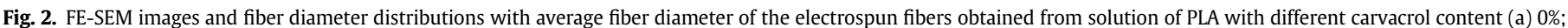
(b) $5 \%$; (c) $10 \%$; (d) $20 \%(\mathrm{w} / \mathrm{w}$ ). 


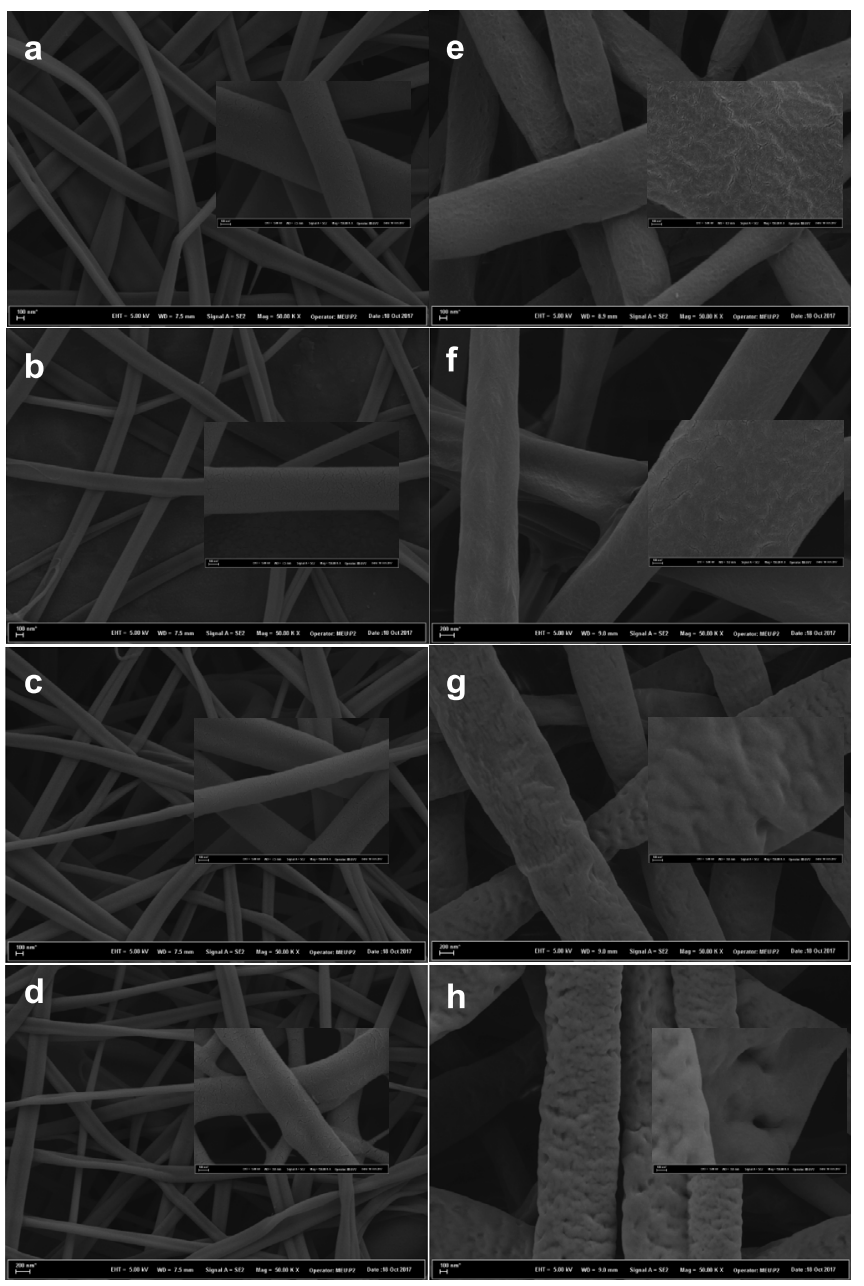

Fig. 3. High magnification FE-SEM images of electrospun fibers from zein (a-d) and PLA (e-h) with different carvacrol content (a-e) $0 \%$; (b-f) $5 \%$; (c-g) $10 \%$; (d-h) $20 \%$ $(\mathrm{w} / \mathrm{w})$.

degradation profile of zein fibers. The addition of carvacrol might act as natural plasticizer and interrupt interactions between zein molecules. Therefore, the significant improvement of thermal stability was not observed for the carvacrol loaded zein fibers. Arrieta et al. (2013) observed that the incorporation of carvacrol did not influence significantly thermal stability of calcium caseinate films but the carvacrol added sodium caseinate films had lower thermal stability. The reduction in thermal stability was explained by the decrease in the number of protein-protein bonds due to the presence of carvacrol in the formulation.

The TGA curves of neat PLA and carvacrol loaded PLA fibers are given in Fig. 5b. The neat PLA fibers showed two stages of weight loss. The initial weight loss (2.3\%) occurred below $80^{\circ} \mathrm{C}$ was attributed to the remaining solvent in the PLA fibers. The main weight loss appeared between $220^{\circ} \mathrm{C}$ and $355^{\circ} \mathrm{C}$ with degradation peak at around $346^{\circ} \mathrm{C}$, which is consistent with the study of Gonçalves, da Silva, Picciani, and Dias (2015) who attributed this degradation to chain scission. The TGA curves for PLA fibers with carvacrol also showed two weight loss stages. Unlike neat PLA fiber, the weight loss below $80^{\circ} \mathrm{C}$ was not observed for carvacrol loaded PLA fibers. This result indicated that there was no remaining solvent in the carvacrol loaded fiber samples. The first stage of weight loss was found to be about 4.4, 6.8 and $11.5 \%$ for PLA fibers at 5, 10 and $20 \%$ carvacrol level, respectively. This weight loss was due to
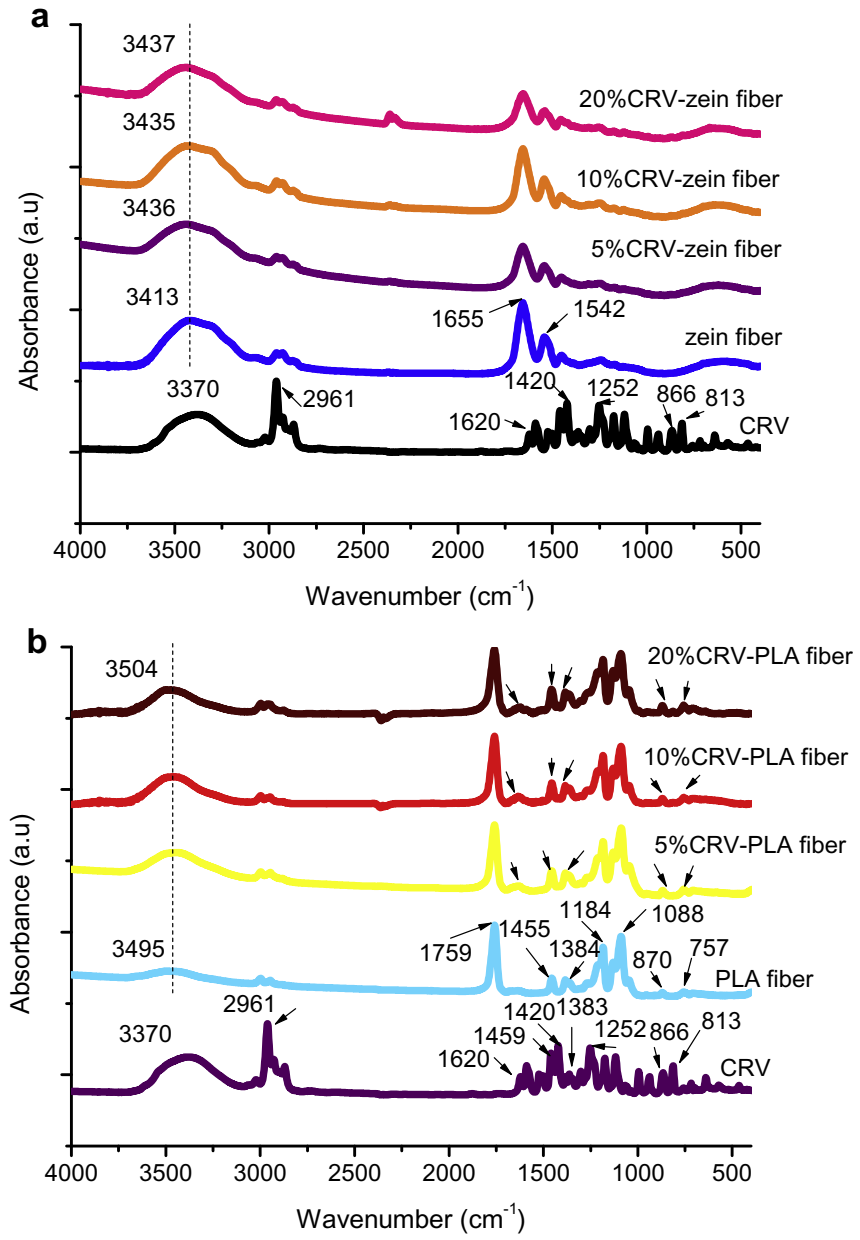

Fig. 4. FTIR spectra of carvacrol, electrospun fibers from (a) zein and (b) PLA with different carvacrol content.

the evaporation of carvacrol incorporated in the blends. The major weight loss occurred in the range of $267-392{ }^{\circ} \mathrm{C}, 273-387^{\circ} \mathrm{C}$ and $267-391{ }^{\circ} \mathrm{C}$ for 5,10 and $20 \%$ carvacrol level. This weight loss indicates thermal decomposition of PLA. The degradation peak temperature of PLA fibers shifted from $346^{\circ} \mathrm{C}$ to 377,376 and $377^{\circ} \mathrm{C}$ for PLA fibers at 5, 10 and 20\% carvacrol when compared to neat PLA fiber. The present results showed that the decomposition temperature of carvacrol loaded PLA fibers is higher than that of neat PLA fibers. Therefore, the addition of carvacrol increased the thermal stability of neat PLA fibers. This increase could be attributed to the interaction between the PLA chains and carvacrol molecules by hydrogen bonding (Alvarado et al., 2018).

\subsection{Release study}

To evaluate release of carvacrol from zein and PLA fibers, the carvacrol peak area obtained from headspace GC-MS was measured at each time interval during releasing of carvacrol until equilibrium reached. Fig. $6 a-b$ shows cumulative release of carvacrol from zein and PLA fibers in terms of peak area as a function of time. The curves indicated that there was an increase in the release of carvacrol when the carvacrol level increased in the fiber samples. An initial burst release followed by sustained release of carvacrol was observed for zein fibers at 5, 10 and 20\% carvacrol level. In the case of PLA fibers, the low sustained release of carvacrol occurred at 5 and 10\% carvacrol level. However, the release profile of PLA fibers 

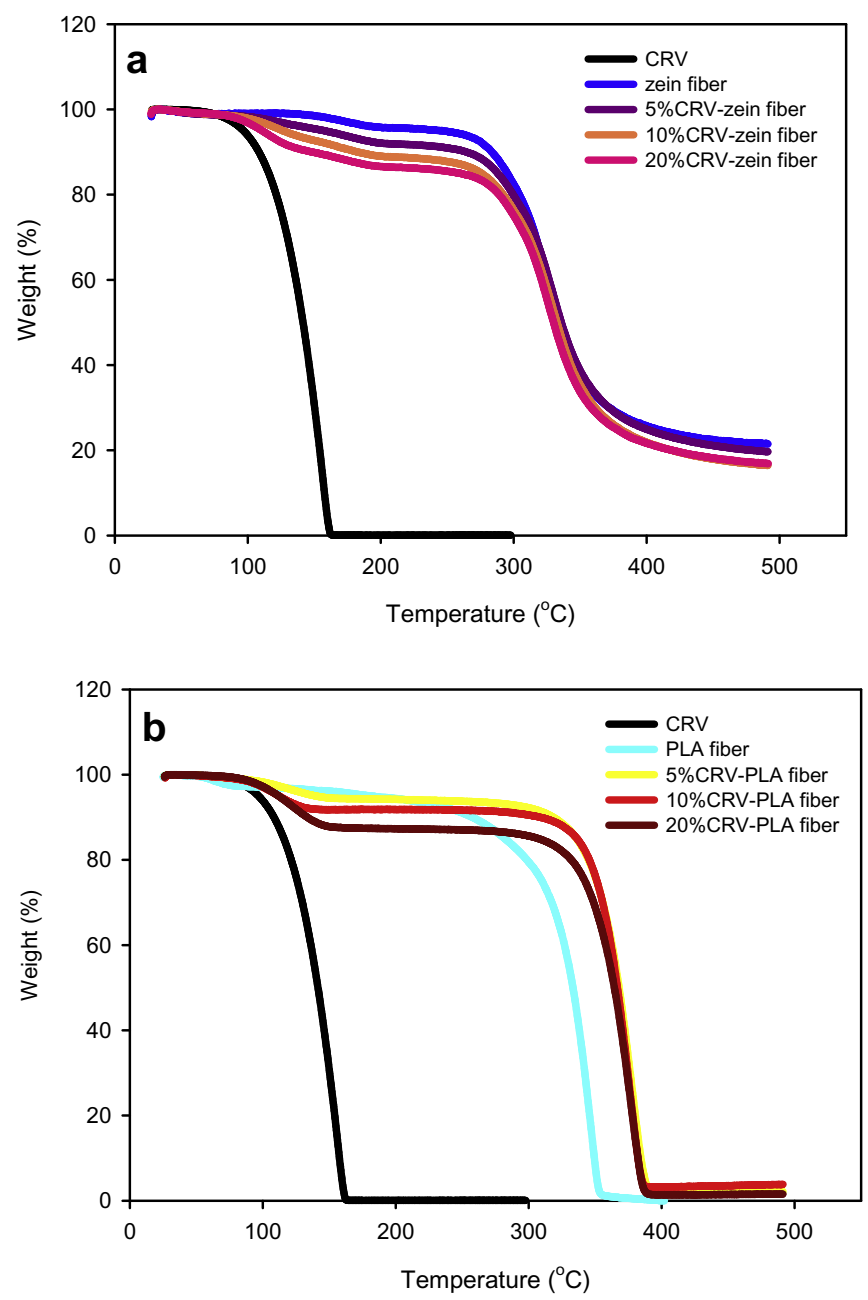

Fig. 5. TGA thermograms of carvacrol, electrospun fibers from (a) zein and (b) PLA with different carvacrol content.

at $20 \%$ carvacrol showed the initial burst effect followed by sustained release. The difference in release profile of PLA fibers was probably due to the morphology and size of fibers obtained at different carvacrol contents (Fig. $3 e-h$ ). The release rate of antimicrobial compound is a critical factor for maintaining food quality and safety. If the release rate of the antimicrobial agent is very slow, microorganisms can grow before antimicrobial compound is released. On the other hand, if the release rate of antimicrobial compound is too fast, the released antimicrobial agent may diffuse into food and may not be available at the food surface to inhibit microbial growth (Marathe, 2008; Uz \& Altınkaya, 2011). Therefore, the controlled release of active agents where an initial relatively higher antimicrobial agent release followed by a slower sustained release may be necessary to maintain its minimum inhibitory concentration on the food surfaces.

The amount of released carvacrol from zein fibers at equilibrium were lower than that released from PLA fibers. As seen from the TGA results, the amount of carvacrol is different from each other in carvacrol containing zein and PLA fibers. Therefore, lower release of zein fibers as compared to PLA fibers might be due to lower amount of carvacrol found in zein fibers as compared to PLA. In addition, zein fibers with and without carvacrol had ribbon morphologies with smooth surfaces (Fig. 3a-d). On the other hand, an increase in carvacrol content from 5 to $20 \%$ in PLA solutions resulted in wrinkles and deep pits on the surfaces of PLA fibers (Fig. $3 f-h$ ).
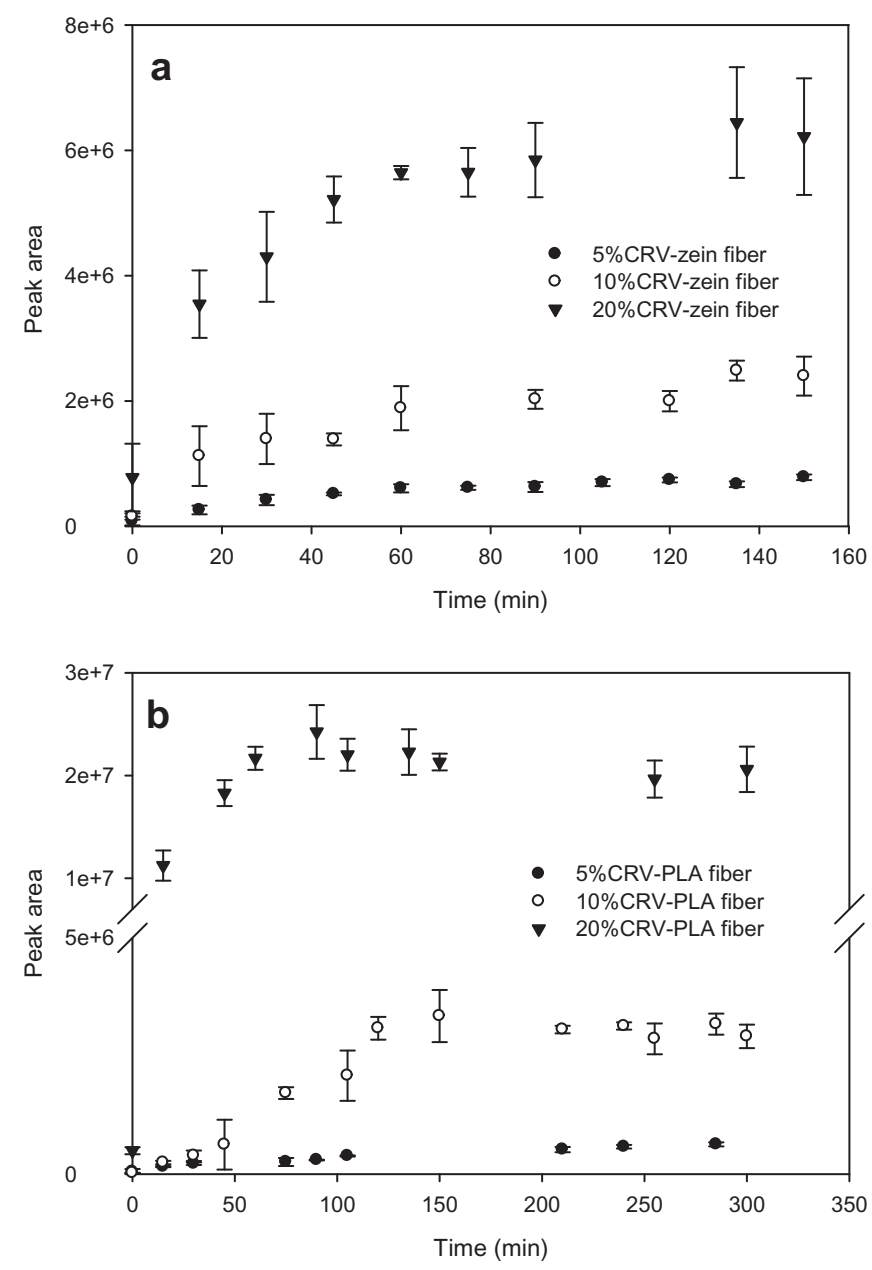

Fig. 6. Cumulative release of carvacrol from electrospun (a) zein and (b) PLA fibers with different carvacrol content.

Therefore, these wrinkles and pits provided much greater surface area per unit volume or mass of materials and hence affected release of carvacrol from PLA fibers (Yang, Zha, Yu, \& Liu, 2013).

The release mechanism of carvacrol from zein and PLA fibers was further investigated using Eq. (3):

$\frac{A_{t}}{A_{\infty}}=k t^{n}$

where $A_{t} / A_{\infty}$, a dimensionless quantity, was calculated by peak area of the released carvacrol at time $t\left(A_{t}\right)$ and peak area of released carvacrol at equilibrium $(A \infty) ; \mathrm{k}$ is the rate constant and $\mathrm{n}$ is the diffusion exponent (Keawchaoon \& Yoksan, 2011; Sangsuwan, Rattanapanone, Auras, Harte, \& Rachtanapun, 2009). The diffusion exponent $n \leq 0.5$ indicates a Fickian diffusion, while $n$ in the range of 0.5 and 1 indicates a non-Fickian (anomalous) diffusion (Sangsuwan et al., 2009). Release kinetic parameters $n$ and $k$ values for carvacrol loaded zein and PLA fibers are given in Table 2. The model gives a good prediction of experimental data with a coefficient of determination $\left(R^{2}\right)$ in the range of $0.951-0.987$. The $n$ values of zein fibers decreased from 0.380 to 0.235 when the carvacrol content increased from 5 to 20\%. A decrease in diffusion exponent $n$ of gallic acid loaded zein fibers with the increase of gallic acid contents was previously reported by Neo et al. (2013b). The $n$ value lower than 0.5 suggested that release of carvacrol from zein fibers showed a typical Fickian diffusion mechanism in which 
Table 2

Diffusion exponent (n) and rate constant (k) for released carvacrol from electrospun zein and PLA fibers at different carvacrol content.

\begin{tabular}{lllll}
\hline Polymer & $\begin{array}{l}\text { Carvacrol content } \\
(\%, \mathrm{w} / \mathrm{w})\end{array}$ & $\mathrm{n}$ & $\begin{array}{l}\mathrm{k} \\
\left(\mathrm{min}^{-\mathrm{n}}\right)\end{array}$ & $\mathrm{R}^{2}$ \\
\hline Zein & 5 & 0.380 & 0.148 & 0.951 \\
& 10 & 0.348 & 0.174 & 0.961 \\
& 20 & 0.235 & 0.325 & 0.959 \\
PLA & 5 & 0.538 & 0.047 & 0.972 \\
& 10 & 0.767 & 0.017 & 0.959 \\
& 20 & 0.289 & 0.273 & 0.987 \\
\hline
\end{tabular}

release caused by the concentration gradient between fibers and releasing medium (Lu, Wang, Li, Qiu, \& Wei, 2017). The values of $n$ were 0.538 and 0.767 for PLA fibers at 5 and $10 \mathrm{wt} \%$ carvacrol content. The release of carvacrol from PLA fibers at 5 and 10\% carvacrol level showed a non-Fickian diffusion mechanism as indicated by a value of $n$. The diffusion exponent of PLA fibers at $20 \%$ carvacrol was 0.289 , suggesting that release of carvacrol was controlled by the Fickian diffusion.

\subsection{Antioxidant activity}

The antioxidant activity of carvacrol loaded zein and PLA fibers was tested by DPPH assay. The DPPH radical scavenging activity is based on the reduction of DPPH in the presence of an H-donating antioxidant into yellow colored substance. The antioxidant capacities of zein and PLA fibers at different carvacrol contents are shown Fig. 7. The antioxidant activity of carvacrol loaded zein fibers ranged from 62 to 75\%, while antioxidant capacity of PLA fibers varied from 53 to $65 \%$ for $5-20 \%$ carvacrol content. The pure zein and PLA fibers also showed 38 and 9\% radical scavenging activity on DPPH. First, the inhibition of pure zein and PLA fibers may be related with the absorption of DPPH solution due to the high surface area of the nanofibers providing more contact with medium (Aytac, Kusku, Durgun, \& Uyar, 2016). Moreover, $\boldsymbol{\alpha}$-zein in pure zein (Li et al., 2013; Zhang, Luo, \& Wang, 2011) and other antioxidants in PLA structure (Iniguez-Franco et al., 2012) contributed to the inherent antioxidant activity of pure zein and PLA fiber. Results showed that DPPH scavenging activity of the both zein and PLA fibers increased as carvacrol content increased (Fig. 7). However, this increase in DPPH activity was only significant $(\mathrm{p}<0.05)$ for zein fibers at $20 \%$ carvacrol level. No significant difference $(p>0.05)$ in antioxidant activity was observed between zein fibers with 5 and $10 \%$ carvacrol.

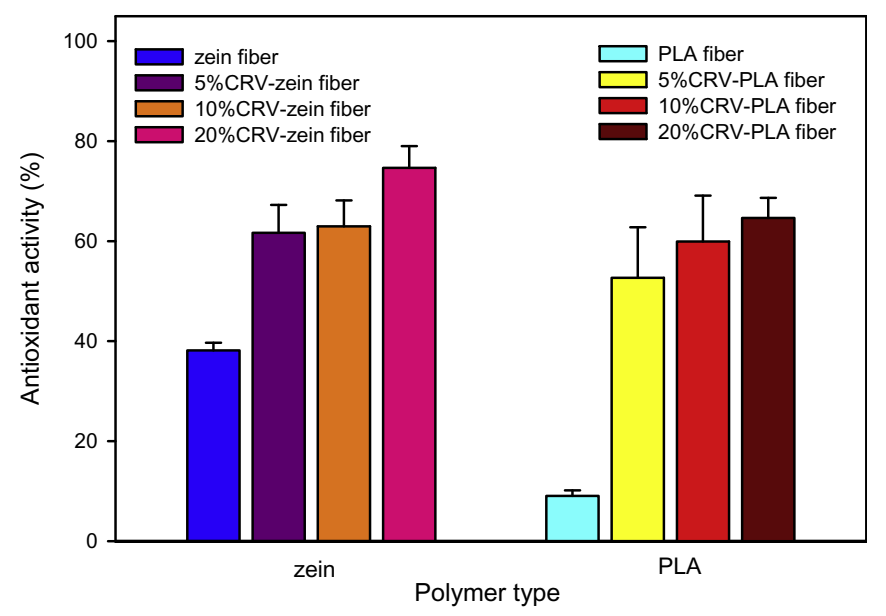

Fig. 7. Antioxidant activity (\%) of electrospun fibers of zein and PLA with different carvacrol content.
According to the results of release study, the amount of carvacrol released from PLA fibers showed significant differences depending on the carvacrol concentration. However, the antioxidant activities of PLA fibers were not significant $(p>0.05)$ at 5, 10 and 20\% carvacrol content. This may be result of small amount of carvacrol extraction by methanol due to the interaction of carvacrol and PLA formed by hydrogen bonding (Alvarado et al., 2018). The zein fiber with 20\% carvacrol exhibited the highest antioxidant activity, 75\% owing to the greater contribution of pure zein in antioxidant activity when compared to pure PLA. On the other hand, PLA fiber with 5\% carvacrol had the lowest antioxidant activity, 53\% (Fig. 7). The results indicated that carvacrol incorporated samples showed antioxidant capacity. Therefore, they may have potential uses as an antioxidant fibrous film in food and packaging applications.

\subsection{Study of effectiveness of the fibers to preserve bread}

The shelf life of bread without preservatives has been reported as around 3-4 days at ambient temperature (Jideani \& Vogt, 2016). Not only microbial growth but also firming of bread limit the shelf life of bread. Refrigeration and addition of preservatives are common methods to extend shelf life of bread. However, staling of bread occurs under refrigeration temperature. There is an increasing consumer concerns about preservatives nowadays. Therefore, there is need to be found a new route to extend shelf life of bread without preservatives. To this end, preliminary tests of zein and PLA fibers with and without carvacrol were performed on whole wheat breads to evaluate their ability to preserve bread samples.

Fig. 8a shows the appearance of bread samples after 7 days of storage at $25^{\circ} \mathrm{C}$. The effect of carvacrol loaded fibers on growth inhibition rate of aerobic mesophilic bacteria and mold and yeast is given in Fig. 8b. The growth of microorganism was easily seen in bread samples with pure zein and PLA fibers. On the other hand, the reduction of microbial growth was observed visually in bread samples with $5 \%$ carvacrol of both zein and PLA fibers. It was noticed that no visual evidence of microbial growth was observed for bread sample with PLA fiber at 10\% carvacrol but bread sample with zein fiber at $10 \%$ carvacrol had mold growth. In the case of bread samples packed with fibers at $20 \%$ carvacrol, no microbial growth was observed visually (Fig. 8a).

The inhibition of both aerobic mesophilic bacteria and mold and yeast growth in bread samples increased as the carvacrol content increased in the both zein and PLA fibers (Fig. 8b). These results confirm what have been shown in the pictures of bread samples in Fig. 8a. Zein fibers at 20\% carvacrol level exhibited $87.6 \%$ and $99.6 \%$ inhibition against aerobic bacteria and mold and yeast whereas the rate of growth inhibitions were $87.0 \%$ and $91.3 \%$ for PLA fibers at $20 \%$ carvacrol level, respectively. The release test, which was performed at higher temperatures, has shown that PLA fibers released more amount of carvacrol than zein. However, here both of the fibers exhibited similar antimicrobial activity and the reason behind this situation might be the stronger interaction of carvacrol and PLA, which do not allow carvacrol to be released as much as zein does at $25^{\circ} \mathrm{C}$. The inhibitory effect of carvacrol has been attributed to the alteration of cell membrane permeability by the action of phenols (Ben Arfa, Combes, Preziosi-Belloy, Gontard, \& Chalier, 2006). The antifungal and antimicrobial effect of carvacrol has been shown in different studies (Nostro et al., 2012; Ramos et al., 2013). Results showed that carvacrol loaded fibers are promising alternatives to increase shelf life of whole wheat bread but further investigations are needed to study effect of released carvacrol into headspace on organoleptic properties of bread. 
a

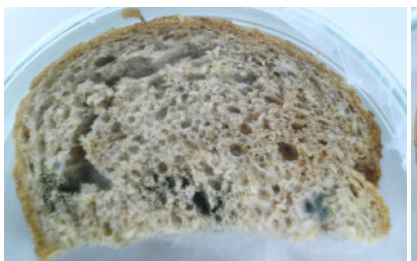

zein fiber

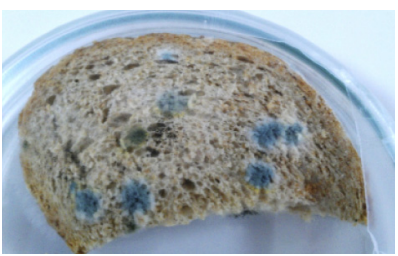

PLA fiber

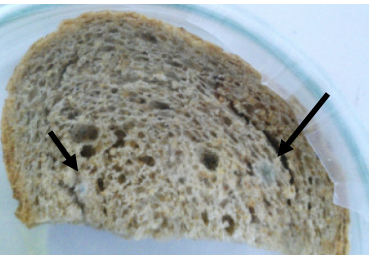

5\%CRV-zein fiber

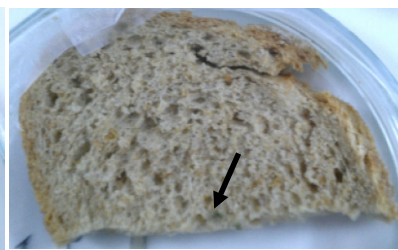

5\%CRV-PLA fiber

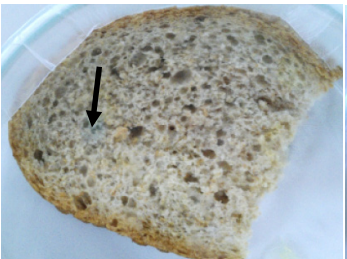

$10 \%$ CRV-zein fiber

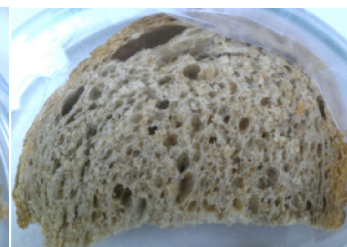

$10 \%$ CRV-PLA fiber

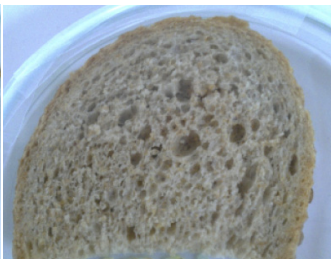

$20 \%$ CRV-zein fiber

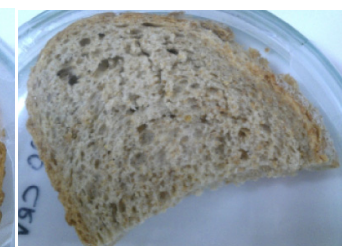

$20 \%$ CRV-PLA fiber

b

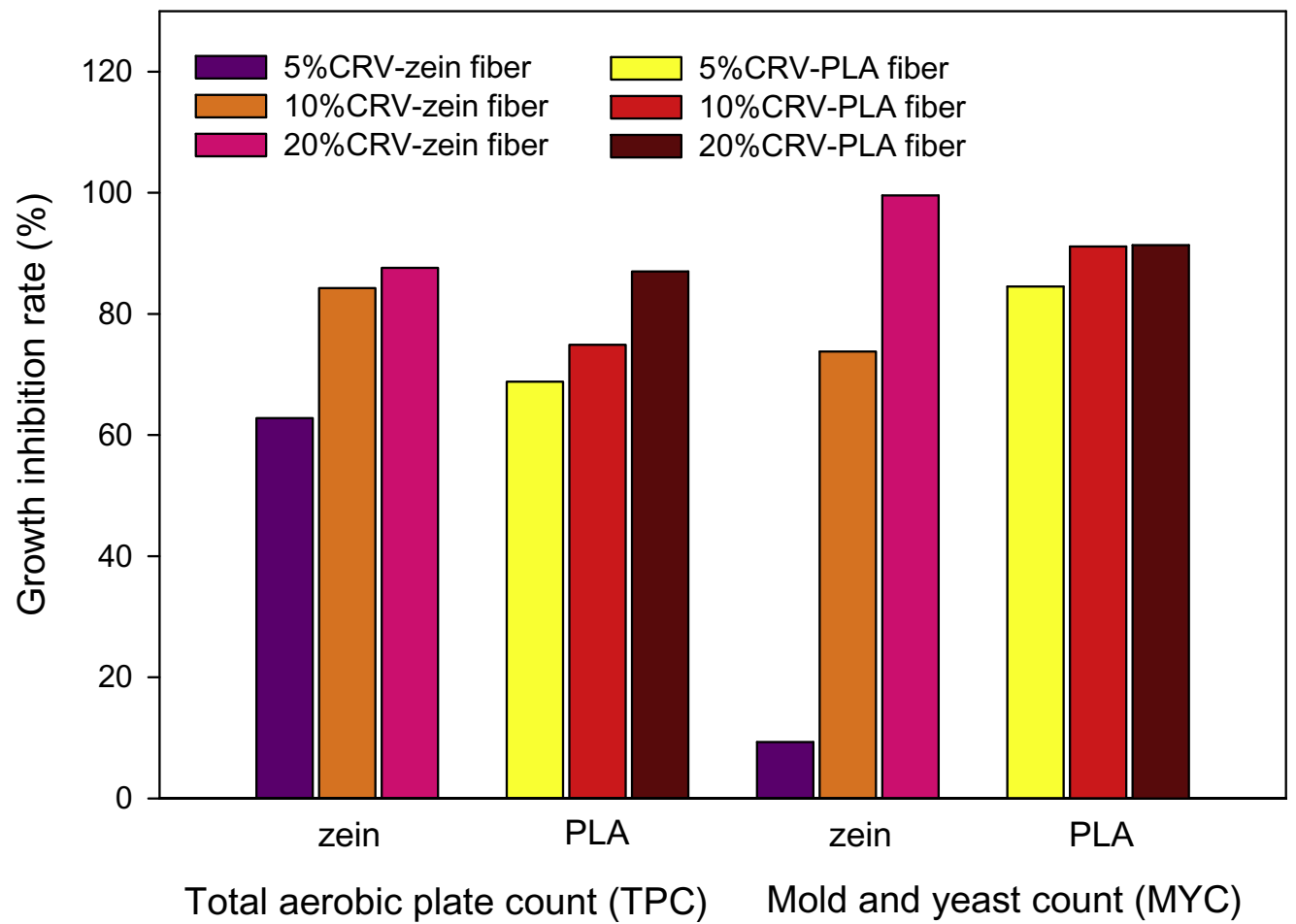

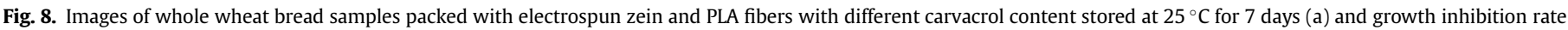
(\%) of aerobic bacteria and mold and yeast (b).

\section{Conclusions}

Results demonstrated that highly volatile carvacrol was successfully encapsulated in electrospun zein and PLA fibers by electrospinning process. SEM images showed that bead free fibers were obtained from zein and PLA polymer solutions containing carvacrol at different concentrations. The incorporation of carvacrol in PLA fibers resulted in an increased thermal stability. In the case of zein, the addition of carvacrol did not influence the thermal stability of zein fibers. The encapsulation of carvacrol in electrospun fibers enhanced sustained release property. The release of carvacrol from electrospun fibers changed depending on the amount of carvacrol and surface morphology of fibers. The release of carvacrol from fibers was Fickian diffusion except for PLA fibers at 5 and $10 \%$ carvacrol content. The composite fibrous zein and PLA films at $20 \%$ carvacrol content inhibited 99.6 and $91.3 \%$ of the growth of mold and yeast. Carvacrol loaded electrospun fibers with its antioxidant and antimicrobial properties can be used to extend shelf life of fresh foods as a new approach of electrospun fibers in food applications. 


\section{Acknowledgements}

\section{Dr. A. Altan acknowledges The Scientific and Technological Research Council of Turkey (TUBITAK) (Project No: 113Z507) for partial funding of this research.}

\section{References}

Alvarado, N., Romero, J. Torres, A., Dicastillo, C. L., Rojas, A., Galotto, M. J., et al. (2018). Supercritical impregnation of thymol in poly(lactic acid) filled with electrospun poly(vinyl alcohol)-cellulose nanocrystals nanofibers: Development an active food packaging material. Journal of Food Engineering, 217, 1-10.

Arcan, I., \& Yemenicioglu, A. (2013). Development of flexible zein-wax composite and zein-fatty acid blend films for controlled release of lysozyme. Food Research International, 51, 208-216.

Armentano, I., Fortunati, E., Burgos, N., Dominici, F., Luzi, F., Fiori, S., et al. (2015). Bio-based PLA_PHB plasticized blend films: Processing and structural characterization. Lebensmittel-Wissenschaft und -Technologie-Food Science and Technology, 64, 980-988.

Arrieta, M. P., Peltzer, M. A., Garrigós, M. C., \& Jiménez, A. (2013). Structure and mechanical properties of sodium and calcium caseinate edible active films with carvacrol. Journal of Food Engineering, 114, 486-494.

Aytac, Z., Keskin, N. O. S., Tekinay, T., \& Uyar, T. (2017). Antioxidant $\alpha$-tocopherol $/ \gamma$ cyclodextrin-inclusion complex encapsulated poly(lactic acid) electrospun nanofibrous web for food packaging. Journal of Applied Polymer Science, 134, 44858.

Aytac, Z., Kusku, S. I., Durgun, E., \& Uyar, T. (2016). Encapsulation of gallic acid/ cyclodextrin inclusion complex in electrospun polylactic acid nanofibers: Release behavior and antioxidant activity of gallic acid. Materials Science and Engineering: C, 63, 231-239.

Ben Arfa, A., Combes, S., Preziosi-Belloy, L., Gontard, N., \& Chalier, P. (2006). Antimicrobial activity of carvacrol related to its chemical structure. Letters in Applied Microbiology, 43, 149-154.

Cosme, J. G. L., Silva, V. M., Nunes, R. R. C., \& Picciani, P. H. S. (2016). Development of biobased poly(lactic acid)/epoxidized natural rubber blends processed by electrospinning: Morphological, structural and thermal properties. Materials Sciences and Applications, 7, 210-219.

Dai, R., \& Lim, L.-T. (2015). Release of allyl isothiocyanate from mustard seed meal powder entrapped in electrospun PLA-PEO nonwovens. Food Research International, 77, 467-475.

Drosou, C. G., Krokida, M. K., \& Biliaderis, C. G. (2017). Encapsulation of bioactive compounds through electrospinning/electrospraying and spray drying: A comparative assessment of food-related applications. Drying Technology, 35(2), 139-162.

El-Naggar, M. N., Abdelgawad, A. M., Salas, C., \& Rojas, O. J. (2016). Curdlan in fibers as carriers of tetracycline hydrochloride: Controlled release and antibacterial activity. Carbohydrate Polymers, 154, 194-203.

Fuenmayor, C. A., \& Cosio, M. S. (2016). Encapsulation of antioxidant phenolic compounds in zein ultra-thin fibers via electrospinning. Revista EIA, 12, E13-E26.

Gonçalves, R. P., da Silva, F. F. F., Picciani, P. H. S., \& Dias, M. L. (2015). Morphology and thermal properties of core-shell PVA/PLA ultrafine fibers produced by coaxial electrospinning. Materials Sciences and Applications, 6, 189-199.

Inigguez-Franco, F., Soto-Valdez, H., Peralta, E., Ayala-Zavala, J. F., Auras, R., \& GamezMeza, N. (2012). Antioxidant activity and diffusion of catechin and epicatechin from antioxidant active films made of poly(L-lactic acid). Journal of Agricultural and Food Chemistry, 60, 6515-6523.

Jideani, V. A., \& Vogt, K. (2016). Antimicrobial packaging for extending the shelf life of bread-a review. Critical Reviews in Food Science and Nutrition, 56, 1313-1324.

Kayaci, F., \& Uyar, T. (2012). Encapsulation of vanillin/cyclodextrin inclusion complex in electrospun polyvinyl alcohol (PVA) nanowebs: Prolonged shelf life and high temperature stability. Food Chemistry, 133, 641-649.

Keawchaoon, L., \& Yoksan, R. (2011). Preparation, characterization and in vitro release study of carvacrol-loaded chitosan nanoparticles. Colloids and Surfaces B: Biointerfaces, 84, 163-171.

Khalifa, S. F. G. (2015). Fabrication and characterization of antibacterial herbal drugloaded polylactic acid/cellulose acetate composite nanofibers for wound dressing applications. MS Thesis. American University in Cairo.

Koombhongse, S., Liu, W., \& Reneker, D. H. (2001). Flat polymer ribbons and other shapes by electrospinning. Journal of Polymer Science Part B: Polymer Physics, 39 2598-2606.

Kriegel, C., Kit, K. M., McClements, D. J., \& Weiss, J. (2009). Electrospinning of chitosan-poly(ethylene oxide) blend nanofibers in the presence of micellar surfactant solutions. Polymer, 50, 189-200.

Li, Y., Lim, L. T., \& Kakuda, Y. (2009). Electrospun zein fibers as carriers to stabilize (-)-epigallocatechin gallate. Journal of Food Science, 74, C233-C240.

Liu, W., Huang, C., \& Jin, X. (2015). Electrospinning of grooved polystyrene fibers: Effect of solvent systems. Nanoscale Research Letters, 10, 237-247.

Li, K. K., Yin, S. W., Yin, Y. C., Tang, C. H., Yang, X. Q., \& Wen, S. H. (2013). Preparation of water-soluble antimicrobial zein nanoparticles by a modified antisolvent approach and their characterization. Journal of Food Engineering, 119, 343-352.
Lopes, F. A., Soares, N. F. F., Lopes, C. C. P., Silva, W. A., \& Júnior, E. A. A. (2014) Conservation of bakery products through cinnamaldehyde antimicrobial films. Packaging Technology and Science, 27, 293-302.

López, P., Sáncez, C., Battle, R., \& Nerín, C. (2007). Development of flexible antimicrobial films using essential oils as active agents. Journal of Agricultural and Food Chemistry, 55, 8814-8824.

Lu, H., Wang, Q., Li, G., Qiu, Y., \& Wei, Q. (2017). Electrospun water-stable zein/ethyl cellulose composite nanofiber and its drug release properties. Materials Science and Engineering: C, 74, 86-93.

Marathe, R. (2008). Development of controlled release antimicrobial films from low methoxyl pectin. Master of Science, Graduate School-New Brunswick Rutgers. New Brunswick, New Jersey: The State University of New Jersey.

Neo, Y. P., Ray, S., Easteal, A. J., Nikolaidis, M. G., \& Quek, S. Y. (2012). Influence of solution and processing parameters towards the fabrication of electrospun zein fibers with sub-micron diameter. Journal of Food Engineering, 109, 645-651.

Neo, Y. P., Ray, S., Jin, J., Gizdavic-Nikolaidis, M., Nieuwoudt, M. K., Liu, D., et al. (2013). Encapsulation of food grade antioxidant in natural biopolymer by electrospinning technique: A physicochemical study based on zein-gallic acid system. Food Chemistry, 136, 1013-1021.

Neo, Y. P., Swift, S., Ray, S., Gizdavic-Nikolaidis, M., Jin, J., \& Perera, C. O. (2013) Evaluation of gallic acid loaded zein sub-micron electrospun fibre mats as novel active packaging materials. Food Chemistry, 141, 3192-3200.

Nostro, A., Scaffaro, R., D’Arrigo, M., Botta, L., Filocamo, A., Marino, A., et al. (2012). Study on carvacrol and cinnamaldehyde polymeric films: Mechanical properties, release kinetics and antibacterial and antibiofilm activities. Applied Microbiology and Biotechnology, 96, 1029-1038.

Oliveira, J. E., Mattoso, L. H. C., Orts, W. J., \& Medeiros, E. S. (2013). Structural and morphological characterization of micro and nanofibers produced by electrospinning and solution blow spinning: A comparative study. Advances in Materials Science and Engineering, 14. Article ID 409572.

Phiriyawirut, M., \& Phaechamud, T. (2012). Gallic acid-loaded cellulose acetate electrospun nanofibers: Thermal properties, mechanical properties, and drug release behavior. Journal of Polymer Chemistry, 2, 21-29.

Quintero, R. I., Rodriguez, F., Bruna, J., Guarda, A., \& Galotto, M. J. (2013). Cellulose acetate butyrate nanocomposites with antimicrobial properties for food packaging. Packaging Technology and Science, 26, 249-265.

Ramos, M., Beltran, A., Valdes, A., Peltzer, M. A., Jiménez, A., Garrigós, M. C., et al. (2013). Carvacrol and thymol for fresh food packaging. Journal of Bioequivalence \& Bioavailability, 5, 154-160.

Ramos, M., Jiménez, A., Peltzer, M., \& Garrigós, M. C. (2012). Characterization and antimicrobial activity studies of polypropylene films with carvacrol and thymol for active packaging. Journal of Food Engineering, 109, 513-519.

Rhim, J. W. (2013). Effect of PLA lamination on performance characteristics of agar к-carrageenan/clay bio-nanocomposite film. Food Research International, 51, 714-722.

Sangsuwan, J., Rattanapanone, N., Auras, R. A., Harte, B. R., \& Rachtanapun, P. (2009). Factors affecting migration of vanillin from chitosan/methyl cellulose films. Journal of Food Science, 74, C549-C555.

Shukla, R., \& Cheryan, M. (2001). Zein: The industrial protein from corn. Industria Crops and Products, 13, 171-192.

Talens, P., Fabra, M. J., \& Chiralt, A. (2011). Properties, applications and current development of edible polysaccharide films and coatings. In C. E. Jones (Ed.) Encyclopedia of polymer research (pp. 857-889). New York: Nova Science Publishers, Inc.

Torres-Giner, S., Gimenez, E., \& Lagaron, J. M. (2008). Characterization of the morphology and thermal properties of Zein Prolamine nanostructures obtained by electrospinning. Food Hydrocolloids, 22, 601-614.

Torres-Giner, S., Ocio, M. J., \& Lagaron, J. M. (2009). Novel antimicrobial ultrathin structures of zein/chitosan blends obtained by electrospinning. Carbohydrate Polymers, 77, 261-266.

Uyar, T., \& Kny, E. (2017). Electrospun materials for tissue engineering and biomedical applications: Research, design and commercialization. Elsevier, Woodhead Publishing Series in Biomaterials.

Uz, M., \& Altınkaya, S. A. (2011). Development of mono and multilayer antimicrobial food packaging materials for controlled release of potassium sorbate. LWT-Food Science and Technology, 44, 2302-2309.

Vega-Lugo, A.-C., \& Lim, L.-T. (2009). Controlled release of allyl isothiocyanate using soy protein and poly(lactic acid) electrospun fibers. Food Research International, 42, 933-940.

Vermeiren, L., Devlieghere, F., van Beest, M., de Kruijf, N., \& Debevere, J. (1999) Developments in the active packaging of foods. Trends in Food Science \& Technology, 10, 77-86.

Wang, Y., \& Chen, L. (2012). Electrospinning of prolamin proteins in acetic acid: The effects of protein conformation and aggregation in solution. Molecular Materials and Engineering, 297, 902-913.

Wen, P., Zhu, D.-H., Wu, H., Zong, M.-H., Jing, Y.-R., \& Han, S.-Y. (2016). Encapsulation of cinnamon essential oil in electrospun nanofibrous film for active food packaging. Food Control, 59, 366-376.

Wongsasulak, S., Tongsin, P., Intasanta, N., \& Yoovidhya, T. (2010). Effect of glycerol on solution properties governing morphology, glass transition temperature, and tensile properties of electrospun zein film. Journal of Applied Polymer Science, 118, 910-919.

Wu, Y., Luo, Y., \& Wang, Q. (2012). Antioxidant and antimicrobial properties of essential oils encapsulated in zein nanoparticles prepared by liquid-liquid dispersion method. LWT-Food Science and Technology, 48, 283-290. 
Yang, J.-M., Zha, L.-S., Yu, D.-G., \& Liu, J. (2013). Coaxial electrospinning with acetic acid for preparing ferulic acid/zein composite fibers with improved drug release profiles. Colloids and Surfaces B: Biointerfaces, 102, 737-743.

Zhang, W., Huang, C., Kusmartseva, O., Thomas, N. L., \& Mele, E. (2017). Electrospinning of polylactic acid fibres containing tea tree and manuka oil. Reactive and Functional Polymers, 117, 106-111.

Zhang, B., Luo, Y., \& Wang, O. (2011). Effect of acid and base treatments on structural, rheological, and antioxidant properties of $\alpha$-zein. Food Chemistry, 59, $7393-7404$. 